\title{
Ship Classification with High Resolution TerraSAR-X Imagery Based on Analytic Hierarchy Process
}

\author{
Zhi Zhao, ${ }^{1}$ Kefeng Ji, ${ }^{1}$ Xiangwei Xing, ${ }^{1}$ Wenting Chen, ${ }^{2}$ and Huanxin Zou ${ }^{1}$ \\ ${ }^{1}$ College of Electronic Science and Engineering, National University of Defense Technology, Changsha, Hunan 410073, China \\ ${ }^{2}$ People's Liberation Army 75711, Guangzhou, Guangdong 510515, China \\ Correspondence should be addressed to Zhi Zhao; zhaozhi_nudt@yahoo.com
}

Received 26 June 2013; Accepted 28 October 2013

Academic Editor: Matteo Pastorino

Copyright (c) 2013 Zhi Zhao et al. This is an open access article distributed under the Creative Commons Attribution License, which permits unrestricted use, distribution, and reproduction in any medium, provided the original work is properly cited.

\begin{abstract}
Ship surveillance using space-borne synthetic aperture radar (SAR), taking advantages of high resolution over wide swaths and all-weather working capability, has attracted worldwide attention. Recent activity in this field has concentrated mainly on the study of ship detection, but the classification is largely still open. In this paper, we propose a novel ship classification scheme based on analytic hierarchy process (AHP) in order to achieve better performance. The main idea is to apply AHP on both feature selection and classification decision. On one hand, the AHP based feature selection constructs a selection decision problem based on several feature evaluation measures (e.g., discriminability, stability, and information measure) and provides objective criteria to make comprehensive decisions for their combinations quantitatively. On the other hand, we take the selected feature sets as the input of KNN classifiers and fuse the multiple classification results based on AHP, in which the feature sets' confidence is taken into account when the AHP based classification decision is made. We analyze the proposed classification scheme and demonstrate its results on a ship dataset that comes from TerraSAR-X SAR images.
\end{abstract}

\section{Introduction}

With the rapid development of worldwide cooperation in ocean economics and military affairs, ship surveillance has attracted many countries' attention. Among various sensors, space-borne synthetic aperture radar (SAR) is considered to be an operational choice for its high resolution over wide swaths and all-weather working capability. Ship surveillance based on space-borne SAR has been widely applied to ocean traffic monitoring, environmental protection, antipiracy operation [1], and so forth. There exist many maritime surveillance systems and projects including ocean monitoring workstation (OMW), IMPAST (improving fisheries monitoring through integrating passive and active satellite based technologies), and DECLIMS (detection and classification of marine traffic form space) [2]. Ship detection in spaceborne SAR $[3,4]$ has been investigated with great progress. Although SAR automatic target recognition (ATR) techniques [5-8] have been investigated a lot, ship classification still has a long way to go. With the rapid development of space-borne SAR, the research on ship classification using high resolution SAR imagery attracts much attention.

In the recent work on SAR ship classification, how to extract features that describes ship accurately and effectively is emphasized. Knapskog et al. [9] investigated the classification of ships based on 3D model and the contour feature. Margarit and Mallorqui [10] extracted scattering center feature for ship classification. They also applied localized RCS features to ship classification and used ships' structural information with ENVISAT data [2, 11]. Margarit and Tabasco [12] presented a new ship classification methodology that uses single-pol synthetic aperture radar (SAR) images to categorize targets based on a fuzzy logic decision rule, via radar signature isolation, parametric vector estimation, and decision steps. Premaratne and Safaei [13] extracted superstructure moment invariants feature from ISAR images for ship classification. Pilcher and Khotanzad [14] classified ships by nonlinear joint classifiers based on multiple feature including length, centroid, and peak value features. Touzi et al. [15] described ships based on the permanent symmetric scatters to achieve better 
TABle 1: Proportional measurements.

Importance evaluation

Numerical value

Coequal importance Little importance More importance Strong importance Extreme importance Median value 3 5 7 9 $2,4,6,8$

classification results. Margarit et al. [16] investigated ship scattering in polarimetric SAR for an improved classification under high clutter conditions and evaluated the potentialities of polarimetric ship scattering for classification methods that provide reasonable performance within cluttered scenes. To utilize the polarimetric interferometric SAR capabilities, they combined Pauli CTD with interferometric-based height retrieval to provide an accurate $3 \mathrm{D}$ representation of the ship geometry in terms of a set of persistent polarimetric scatterers [17]. Wu et al. [18] analyzed three types of features from fine quad polarization Radarsat-2 SAR image to characterize the ships and prepare for ship classification. Lakshmi et al. [19] analyzed the polarimetric SAR data for identification and characterization of ships, and the polarimetric signature of interaction of the metallic structure of the ship with water is clearly demarcated for the first time through a particular scattering mechanism representing a dipole scatterer. In the related researches, multifeature is much preferred than single feature and achieves better performance. However, the stateof-the-art feature selection is usually based on the experiences or rough evaluations, and lacks valid comprehensive evaluations.

Feature selection is an essential and fundamental problem for classification. Different feature always leads to various classification performances. To choose an optimal feature subset is significant for classification. There are kinds of feature selection methods (i.e., filter and wrapper). State-ofthe-art evaluation criteria for filter methods [20-22] include discriminability measure, stability measure, and information measure. Single criteria based feature selection can be easily implemented, but it lacks quantitative and objective evaluation for multifeature combination. Therefore, it is necessary to establish objective and comprehensive evaluation criteria for feature selection.

Analytic hierarchy process (AHP) is a structured technique for organizing and analyzing complex decisions. In this paper, we firstly introduce AHP to feature selection. It accounts for the comprehensive performances of multiple evaluation measures and provides valid and objective criteria to make comprehensive decisions for their combinations quantitatively. Moreover, similar to the feature selection procedure, we also introduce AHP to the classification decision to fuse the results of different feature sets. The contribution of the proposed AHP based ship classification scheme lies on two aspects: (1) a feature selection method is derived based on the AHP; (2) a classification decision rule is proposed based on AHP to fuse the classification results of different selected feature sets. The proposed classification scheme has been tested on a ship dataset that comes from high resolution TerraSAR-X SAR images. Preliminary results illustrate that the feature selection method is effective and the classification scheme achieves a ratio of positive classifications around $87.7 \%$.

This paper is organized as follows. Section 2 provides a brief review of the analytic hierarchy process. Section 3 includes the description of the classification scheme, with special concern on the procedure of feature selection and classification decision based on AHP, respectively. Then, Section 4 validates the method with high resolution TerraSAR-X SAR images, including the detail implementation of AHP for feature selection and classification decision. Finally, the paper is concluded in Section 5.

\section{Analytic Hierarchy Process}

Analytic hierarchy process (AHP) [23-30] is a structured technique for organizing and analyzing complex decisions. Since presented by Saaty in the 1970s, it has been applied in a wide variety of decision situations. The AHP provides a comprehensive and rational framework for structuring a decision problem, for representing and quantifying its elements, for relating those elements to overall goals, and for evaluating alternative solutions. When a hierarchy is built, the decisionmakers systematically evaluate various elements by comparing them to one another two at a time, with respect to their impact on an element above them in the hierarchy.

AHP has unique advantages when important elements of the decision are difficult to quantify or compare. It is applicable in the condition that there exists multiple-goal decision on evaluating the schemes based on multiple influencing factors. When AHP is applied, four premises need to be satisfied: the elements on every layer are known and the hierarchy is clear; the elements on the same layer are uncorrelated; the elements on the lowest layer could be quantitated; the relations among different elements on different layers need to be definite. The procedure for the AHP can be summarized as follows.

(i) Build the hierarchy model. Classify the correlative factors into three or more layers: Top layer (the purpose to solve problem); middle layer (the measures and criteria to use, also called strategy layer or criteria layer), whose number could be more than one; bottom layer (the schemes used to resolve problems).

Constitute the pair-wise comparison matrix for the middle layer based on the pair-wise comparison method and 19 comparison measure. Priorities among the elements of the hierarchy are achieved by making a series of judgments based on pair-wise comparisons. When comparing the importance of the $i$ th factor with the $j$ th factor on the middle layer, it is described by the quantitative relative weighting $a_{i j}$ (1 9 and their reciprocals). 1 9 represents different levels for importance; see Table 1. Assume there are $M$ factors to be compared for the middle layer; the pair-wise comparison matrix is $\mathbf{A}_{M \times M}=\left(a_{i j}\right)_{M \times M}$. 
TABLE 2: Standard value of the average random consistency index RI.

\begin{tabular}{lllcccccccc}
\hline Matrix rank number & 1 & 2 & 3 & 4 & 5 & 6 & 7 & 8 & 9 \\
\hline RI & 0 & 0 & 0.58 & 0.90 & 1.12 & 1.24 & 1.32 & 1.41 & 1.45 & 1.49 \\
\hline
\end{tabular}

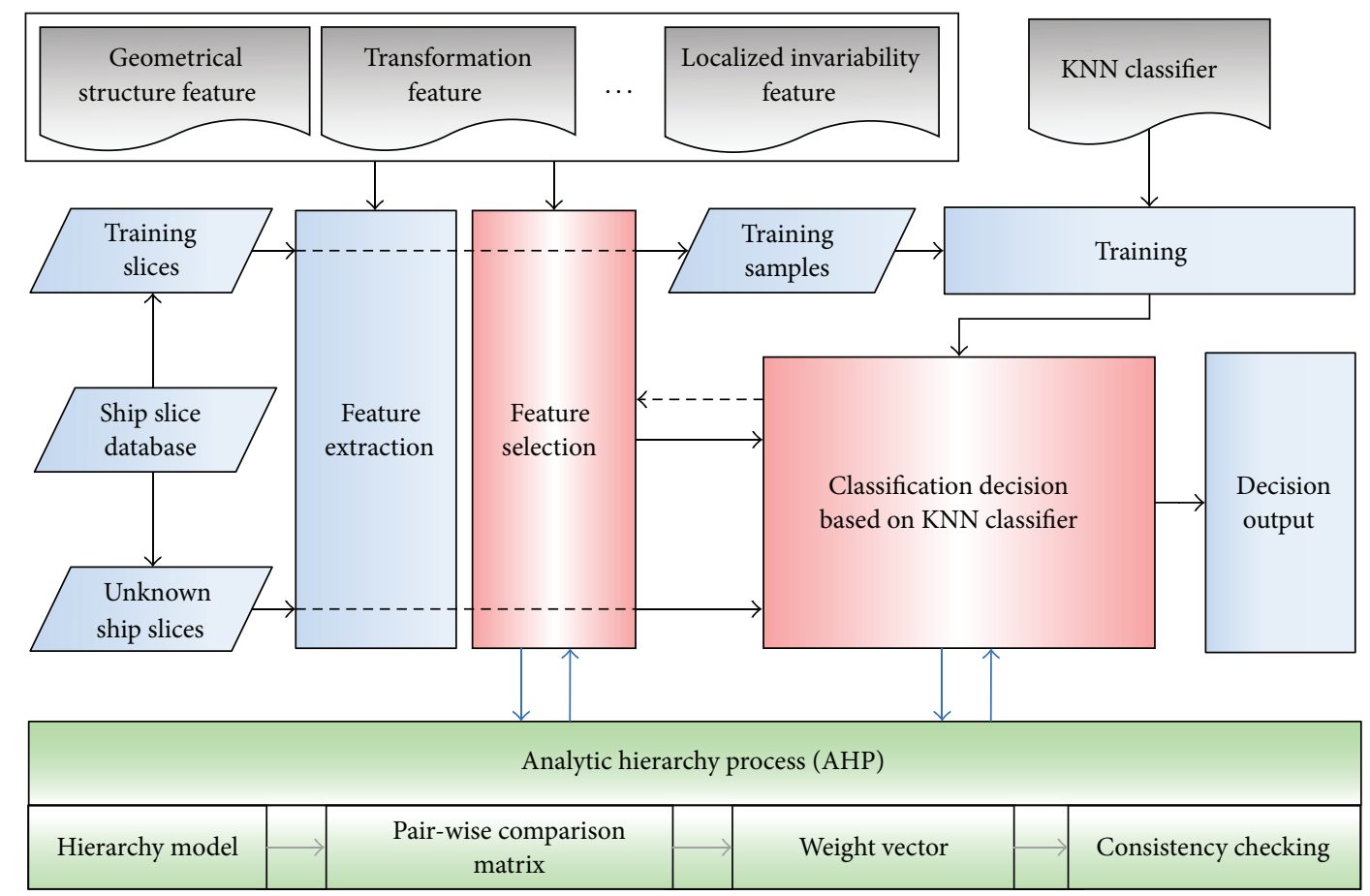

FIGURE 1: Ship classification model.

(ii) Calculate the weight vector of $\mathbf{A}$ and check the consistency of the judgments. Calculate the maximum eigenvalue and corresponding eigenvector of $\mathbf{A}$, and then check the consistency. If the check succeeds, the normalized eigenvector is assigned as the weight vector s. If not, the comparison matrix needs to be constituted again. In detail, the steps are illustrated below. Firstly, calculate the index CI to assess the nonconsistency of pair-wise comparison matrix A:

$$
\mathrm{CI}=\frac{\lambda_{\max }(\mathbf{A})-M}{M-1} .
$$

And, then, calculate the average random consistency index RI. It is acquired by building pair-wise comparison matrix $\mathbf{A}$ randomly to get the average of maximum eigenvalues with large enough random samples. It is validated to be related to matrix rank number $M$ only (see Table 2).

Thirdly, calculate the random consistency ratio CR:

$$
\mathrm{CR}=\frac{\mathrm{CI}}{\mathrm{RI}}
$$

When CR $<0.1$, the consistency of matrix $\mathbf{A}$ is satisfied. Otherwise, A needs to be reconstructed.

(iii) Corresponding to each element of the middle layer, a pair-wise comparison matrix for the bottom layer could be derived. So there are $M$ pair-wise comparison matrix for the bottom layer. Assume that the number of the elements for the bottom layer is $N$; the $N \times N$ pair-wise comparison matrix could be expressed as $\mathbf{B}_{i}(i=1,2, \ldots, M)$. And then, check the consistency of them using (1) and (2). If the check succeeds $(\mathrm{CR}<0.1)$, the maximum eigenvalue and corresponding eigenvector are derived for each of them. The weight vectors are calculated by normalizing the maximum eigenvalue vectors. So there are $x$ weight vectors $\mathbf{v}_{i}(i=1,2, \ldots, M)$, and they could be concatenated into a weight-vector matrix $\mathbf{V}_{M \times N}$.

(iv) Come to a final decision based on the results of this process. There is a need needs to calculate the total scores for every scheme of bottom layer and give the orders of them. The final decision is made based on the order of $\mathbf{s} \cdot \mathbf{V}$.

\section{Ship Classification Based on AHP}

In this section, we present the proposed ship classification scheme, with special concern on the AHP based feature selection and classification decision procedures.

3.1. Classification Scheme. The proposed scheme for ship classification is illustrated in Figure 1. The basic process includes four main parts: feature extraction, feature selection, classifier training and classification decision. The kernel idea of the proposed model is to apply analytic hierarchy process to the feature selection and classification decision. In this paper, the evaluation for feature selection adopts filter measures. Ship classification is mainly based on the single classifier and takes 


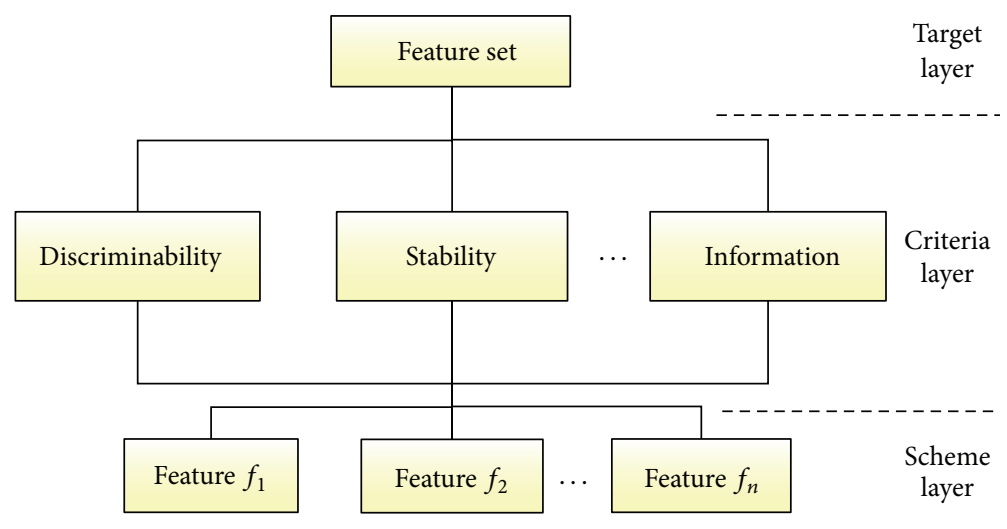

FIGURE 2: Hierarchy model for feature selection.

the KNN classifier as an example to illustrate the problem. It is worth noting that the feature extraction and classifier training have been widely investigated [31-33] and this paper will not pay additional attention to these processes.

3.2. Feature Selection Based on AHP. Features derived from ship slices are used as the input of classifier for ship classification. Considering the same classifier, different feature set always leads to different classification result. The feature selection is necessary to improve the performance. The hierarchy model for feature selection is presented in Figure 2.

Assume that the evaluation measure vector is $\left(e_{1}, e_{2}, \ldots\right.$, $\left.e_{M}\right)$. The weightiness of them could be expressed as $\left(g_{1}, g_{2}\right.$, $\left.\ldots, g_{M}\right)$. The relative weightiness $a_{i j}$ could be expressed as

$$
a_{i j}=\varphi\left(\frac{g_{i}}{g_{j}}\right), \quad i, j \in\{1,2, \ldots, M\},
$$

where $\varphi(\cdot)$ is the mapping from accuracy rate domain $\left\{g_{i} / g_{j}\right\}$ to the discrete weighting domain illustrated in Table 1. So the pair-wise comparison matrix is:

$$
\begin{aligned}
\mathbf{A} & =\left[\begin{array}{ccccc}
1 & a_{12} & a_{13} & \cdots & a_{1 M} \\
a_{21} & 1 & a_{23} & \cdots & a_{2 M} \\
a_{31} & a_{32} & 1 & \cdots & a_{3 M} \\
\vdots & \vdots & \vdots & 1 & \vdots \\
a_{M 1} & a_{M 2} & a_{M 3} & \cdots & 1
\end{array}\right] \\
& =\left(\left[\begin{array}{ccccc}
1 & \frac{g_{1}}{g_{2}} & \frac{g_{1}}{g_{3}} & \cdots & \frac{g_{1}}{g_{M}} \\
\frac{g_{2}}{g_{1}} & 1 & \frac{g_{2}}{g_{3}} & \cdots & \frac{g_{2}}{g_{M}} \\
\frac{g_{3}}{g_{1}} & \frac{g_{2}}{g_{2}} & 1 & \cdots & \frac{g_{3}}{g_{M}} \\
\vdots & \vdots & \vdots & 1 & \vdots \\
\frac{g_{M}}{g_{1}} & \frac{g_{M}}{g_{2}} & \frac{g_{M}}{g_{3}} & \cdots & 1
\end{array}\right]\right.
\end{aligned}
$$

After calculation for the maximum eigenvalue of matrix A, the check of consistency can be performed through comparison between $\mathrm{CR}$ and 0.1. In addition, the maximum eigenvalue vector can also be derived:

$$
\mathbf{s}^{*}=\left(m_{1}, m_{2}, m_{3}, \ldots, m_{M}\right) \text {. }
$$

It needs to be normalized to a standardization vector $\mathbf{s}$ with the conditions that

$$
\begin{gathered}
m_{i}>0, \\
\sum_{i} m_{i}=1 .
\end{gathered}
$$

As to one of the evaluation measures, the pair-wise comparison matrix for the scheme layer is

$$
\mathbf{B}_{i}=\left[\begin{array}{ccccc}
1 & b_{12} & b_{13} & \cdots & b_{1 N} \\
b_{21} & 1 & b_{23} & \cdots & b_{2 N} \\
b_{31} & b_{32} & 1 & \cdots & b_{3 N} \\
\vdots & \vdots & \vdots & 1 & \vdots \\
b_{N 1} & b_{N 2} & b_{N 3} & \cdots & 1
\end{array}\right], \quad i \in\{1,2, \ldots, M\}
$$

The weight vectors of $\left\{\mathbf{B}_{i}, i=1,2,3, \ldots, M\right\}$ can be calculated by the normalization of the maximum eigenvalue vectors:

$$
\begin{gathered}
\mathbf{v}_{1}=\left(x_{1}^{(1)}, x_{2}^{(1)}, x_{3}^{(1)}, \ldots, x_{N}^{(1)}\right) \\
\mathbf{v}_{2}=\left(x_{1}^{(2)}, x_{2}^{(2)}, x_{3}^{(2)}, \ldots, x_{N}^{(2)}\right) \\
\vdots \\
\quad \vdots \\
\mathbf{v}_{M}=\left(x_{1}^{(M)}, x_{2}^{(M)}, x_{3}^{(M)}, \ldots, x_{N}^{(M)}\right) .
\end{gathered}
$$

And then $\left\{\mathbf{v}_{i}, i=1,2, \ldots, M\right\}$ constitutes a matrix $\mathbf{V}$ :

$$
\mathbf{V}=\left(\mathbf{v}_{1}, \mathbf{v}_{2}, \mathbf{v}_{3}, \ldots, \mathbf{v}_{\mathbf{M}}\right)^{T} .
$$

The final decision on feature selection can be made through comparisons of total scores of the features to be chosen:

$$
\operatorname{Sort}(\mathbf{s} \cdot \mathbf{V}) \text {. }
$$




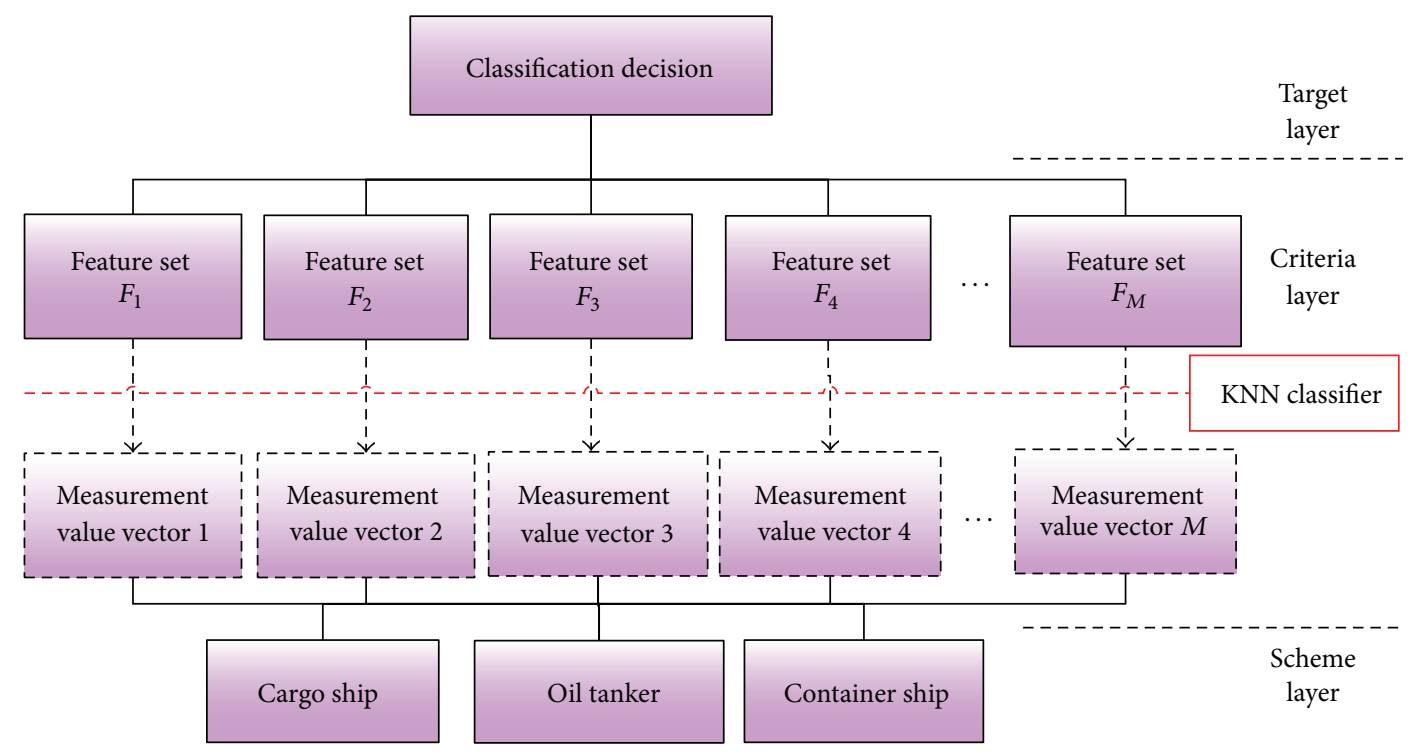

FIGURE 3: Hierarchy model for classification decision based on single classifier.

3.3. Classification Decision Based on AHP. Because the evaluation measures including discriminability, stability, and information are calculated based on certain amount of samples (nonsufficient theoretically), the errors are unavoidable. According to the calculated orders of features, feature selection needs to consider the error tolerance. For example, if the optimal feature set is made up of five features, six or seven features (the former six or seven of the descending order) need to be taken into account, so there are $C_{6}^{5}$ or $C_{7}^{5}$ feature sets $\left\{F_{M}\right\}$ that should be considered.

To set the feature sets derived above as the input for $\mathrm{KNN}$ classifier, respectively, multiple classification measurement value vectors (not the final labels) could be acquired. And then the final classification decision is made using the AHP based comprehensive processing of these measurement value vectors.

Firstly, construct the hierarchy model. When a ship is classified into three kinds (e.g., cargo ship, oil tanker, and container ship) based on the KNN classifier, feature sets $\left\{F_{M}\right\}$ make up the evaluation evidence (criteria layer). The hierarchy model is shown in Figure 3. Unlike the feature selection, the pair-wise comparison matrix for the scheme layer is constructed based on the classification measurement value vectors of a feature set, neither the value of the feature set itself nor the final classification labels (cargo ship, oil tanker, or container ship).

Secondly, the pair-wise comparison matrix can then be constructed based on the comparisons of different feature sets' contributions. Due to the stochastic errors, the feature sets could be considered with the same importance (the comparison matrix of all ones). Another method to determine the weighting among the feature sets is based on the feature's priority in the feature set. For example, there are two feature sets $\left\{f_{1}, f_{2}, f_{3}, f_{4}, f_{5}\right\}$ and $\left\{f_{1}, f_{2}, f_{3}, f_{4}, f_{6}\right\}$; assume that the feature priorities are $f_{1}>f_{2}>f_{3}>f_{4}>f_{5}>f_{6}$; then the first feature set is judged to be prior to the second. The priorities for multiple feature sets could be transformed into their confidence, so $a_{i j}$ is the confidence ratio between feature sets $i$ and $j$. The comparison matrix $\mathbf{A}_{M \times M}$ could be constituted. After calculation for the maximum eigenvalue of matrix $\mathbf{A}_{M \times M}$, the check of consistency can be performed through comparison between $\mathrm{CR}$ and 0.1. In addition, the maximum eigenvalue vector can also be derived:

$$
\mathbf{s}^{*}=\left(m_{1}, m_{2}, m_{3} \cdots m_{M}\right) \text {. }
$$

It also needs to be transformed into a standardization vector s. There are three schemes (type I: cargo ship, type II: oil tanker, and type III: container ship) for classification under every feature set, so the $M$ corresponding pair-wise comparison matrix $\mathbf{B}_{i}, i \in\{1,2, \ldots, M\}$, could be produced:

$$
\text { I } \quad\left[\begin{array}{ccc}
\text { I } & \text { II } & \text { III } \\
\text { III } & b_{12} & b_{13} \\
b_{21} & 1 & b_{23} \\
b_{31} & b_{32} & 1
\end{array}\right] \text {. }
$$

The weight coefficients are calculated by

$$
b_{i j}=\varphi\left(\frac{g_{i}}{g_{j}}\right), \quad i, j \in\{1,2,3\},
$$

where $g_{i}$ is the measurement value of the classifier's output.

Take the KNN classifier [34] as an example; when a ship chip $x$ is classified, the discriminant of the $i$ th type of ship is

$$
d_{i}(x)=k_{i},
$$

where $k_{i}$ is the number of $K$ nearest neighbor samples which belongs to the $i$ th type of ship. So the measurement value is

$$
g_{i}=d_{i}(x)
$$

After checking it consistency, if succeeded, the weight vectors matrix $\mathbf{V}=\left\{\mathbf{v}_{i}^{T}\right\}$ of $\mathbf{B}$ could be calculated. The total 

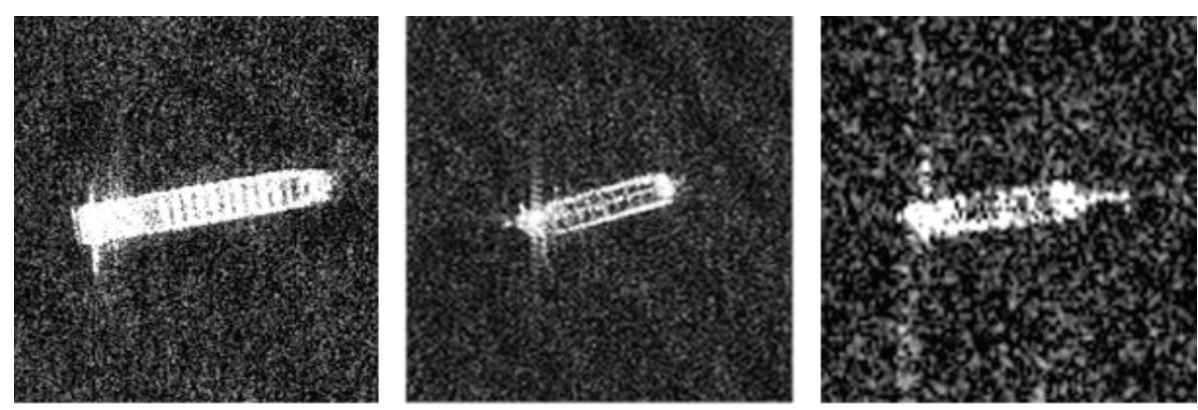

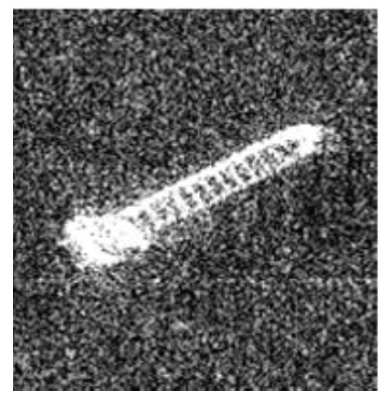

Container ship

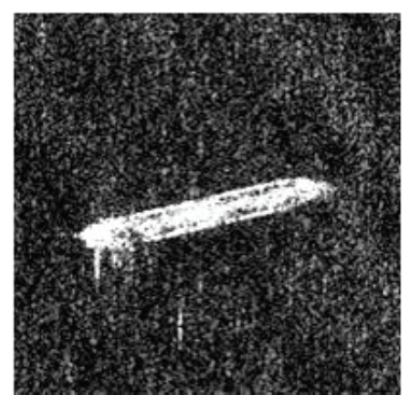

Oil tanker

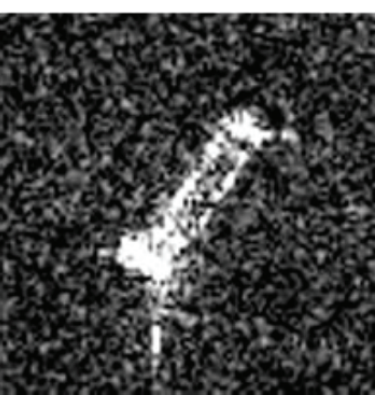

Cargo ship

FIGURE 4: Samples of ship chips.

scores of the three types are calculated by the products of the weight vector of the middle layer pair-wise comparison matrix and the weight vector of the bottom layer pair-wise comparison matrix. The final decision on classification for a single classifier based on multiple feature sets can be made through comparisons of the total scores:

$$
\operatorname{Sort}(\mathbf{s} \cdot \mathbf{V}) \text {. }
$$

\section{Experiments and Analysis}

4.1. Data Set. To validate the proposed methods, the experiments are carried out using TerraSAR-X SAR imagery with VV polarization, azimuth resolution 3.3 meters, and ground range resolution 1.9 meters [35]. Ship chips extracted from the imagery make up the experiment database.

In SAR images, the appearance of ship varies with many factors, such as atmospheric phenomena, image resolution, ship speed, ship orientation, ship size, and the material of the ship. In this paper, the high resolution TerraSAR-X images are captured from the near coastal areas; the sea state is considered as stable. For simplification, the atmospheric effects are neglected. For the cases of nonmetallic or small ship, the ship scattering is not clear and difficult to be identified even by experts. It is a challenging work for the state-of-the-art methods to recognize these kinds of ships. Additionally, the ships with severe smearing and occlusion that result from ship cruising and various orientations are discarded when the data set is constituted. Therefore, we focus on the metallic ships with relative large dimensions and clear appearances on the SAR images.

The number of TerraSAR-X ship chips collected is 286 . Ship classification was firstly done by the human operator. There are 56 oil tanker ships, 160 cargo ships, and 70 container ships, respectively. Figure 4 shows two examples of each kind. We choose $60 \%$ of each kind of ships as the training set and the left $40 \%$ as the test set, whereas the training set includes 96 cargo ship samples, 42 container ship samples, and 34 oil tanker ship samples, and the test set is composed of 64 cargo ship samples, 28 container ship samples, and 22 oil tanker ship samples.

4.2. Feature Selection. In this experiment, 21 features [31-33] were extracted from each ship from the database $(N=21)$. They include length $f_{1}$, width $f_{2}$, length to width ratio $f_{3}$, area $f_{4}$, perimeter $f_{5}$, shape complexity $f_{6}$, centroid $f_{7}$, moment of inertia $f_{8}$, mass $f_{9}$, the mean intensity $f_{10}$, variance coefficient $f_{11}$, weighted-rank fill ratio $f_{12}$, standard deviation $f_{13}$, fractal dimension $f_{14}$, and Hu moment $f_{15} \sim f_{21}$. These features belong to the geometrical structural features and gray intensity statistical features.

In addition, electromagnetic scattering features are also important to ship classification, but they are normally difficult to extract and require much higher image resolution. They increase the information quantity using both the magnitude and phase information of the complex SAR image, relate closely to the ships' scattering characteristics, and reflect the targets' essential information. In this paper, in order to illustrate the problem concisely, we only choose the 21 features presented above.

Three evaluation measures including feature discriminability, feature stability, and BIF (best individual feature) are considered in this experiment. Correlation measure is one of the important measures for feature selection to reduce the redundancy. The paper selects three measures just to illustrate the problem, and actually more measures could be adopted including the correlation measure. The definition of 
the features and the evaluation measures are beyond the scope of this paper, and they can be referenced in the literatures [2022].

Firstly, the comparison matrix for the three evaluation measures could be set according to the experiences. If it is not easy to decide the weighting, it could also be set as all ones (with the same importance). In this paper, it is set by experience. Discriminability (measure I) is considered as the most important one, and stability (measure II) is more important than BIF (measure III). So $M=3$, and $\mathbf{A}$ could be set as

$$
\text { II } \quad\left[\begin{array}{ccc}
\text { I } & \text { II } & \text { III } \\
\text { III } & 3 & 5 \\
\frac{1}{3} & 1 & 3 \\
\frac{1}{5} & \frac{1}{3} & 1
\end{array}\right] \text {. }
$$

And then, the maximum eigenvalue of $\mathbf{A}$ is $\lambda_{\max }(A)=$ 3.0554, CI $=\left(\lambda_{\max }(\mathbf{A})-n\right) /(n-1)=0.0277$. We look up Table 2 to get the value of RI $(\mathrm{RI}=0.58)$, so $\mathrm{CR}=\mathrm{CI} / \mathrm{RI}=$ $0.0478<0.1$, and the consistency of $\mathbf{A}$ is satisfied.

The standardization form of the maximum eigenvector is

$$
\mathbf{s}=[0.6054,0.2915,0.1031]^{T} .
$$

Based on the training samples, the three evaluation measures are calculated and the results are illustrated in Figure 5, respectively.

According to the results above, the comparison matrix for discriminability, stability, and BIF could be derived: $\left.\mathbf{B}_{i}\right|_{21 \times 21}, i \in\{$ discriminability, stability, BIF $\}$. And then, the weighting vectors of them are acquired:

$$
\begin{aligned}
& \mathbf{v}_{\text {discriminaloility }} \\
& =[0.0747,0.0305,0.0765,0.0623,0.0676, \\
& \quad 0.0596,0.0305,0.0623,0.0605,0.0489, \\
& \quad 0.0182,0.0114,0.0279,0.0308,0.0209, \\
& \quad 0.0525,0.0311,0.0605,0.0418,0.0640,0.0676]^{T}, \\
& \mathbf{v}_{\text {stability }} \\
& =[0.0385,0.0200,0.0696,0.0623,0.0197, \\
& \quad 0.0623,0.1026,0.0360,0.0385,0.0238, \\
& \quad 0.0360,0.0360,0.0360,0.0342,0.0281,0.0623, \\
& \quad 0.1026,0.0733,0.0360,0.0623,0.0197]^{T}, \\
& \mathbf{v}_{\text {BIF }} \quad \begin{array}{l}
0.0760,0.0472,0.0553,0.0461,0.0461, \\
=[0.0472,0.0392,0.0461,0.0461,0.0461,
\end{array} \\
& 0.0403,0.0415,0.0449,0.0438,0.0484,0.0484, \\
& 0.0484,0.0472,0.0461,0.0484]^{T} .
\end{aligned}
$$

The total score for each feature is shown in Figure 6.
Thus the descending order of them is $f_{3}>f_{1}>f_{18}>$ $f_{20}>f_{4}>f_{6}>f_{16}>f_{17}>f_{8}>f_{9}>f_{7}>f_{21}>f_{5}>f_{10}>$ $f_{19}>f_{14}>f_{13}>f_{2}>f_{15}>f_{11}>f_{12}$.

It can be concluded that all the geometrical structural features have better performances except feature $f_{2}$ (width). Geometrical structural features relate closely to the ships' size, shape, and structure. In the metallic large ship cases (cargo, oil tanker, and container ship), geometrical structural features perform better theoretically. Three typical types of ships differ from each other in terms of size, shape, and structure, and the results indicate that these features have better performance, especially for feature $f_{3}$ (length to width ratio) which is important for ship classification. Most of the $\mathrm{Hu}$ moment features perform better in the experiment. They describe the characteristics of ships' gray intensity distribution and edge shapes and reflect the ships' essential attributes. Gray intensity statistical features including weighted-rank fill ratio $f_{12}$, standard deviation $f_{13}$, and fractal dimension $f_{14}$ describe the regularity and structure information of the pixels' gray intensity distribution. They are preferable and also widely used in the ship classification; however, the experimental results illustrate that they perform worse than the geometrical structural features as a whole for ship classification of these three types.

Feature selection could be implemented according to the results above. The features with higher scores or orders should be considered to make up the optimal feature set, but the number is still unknown. To derive it, 20 feature sets are constructed based on the derived order and ship classification based on the test set is carried out to evaluate the accuracy. The first set is $\left\{f_{3}, f_{1}\right\}$, adding one feature based on the order once a time, so the second one is $\left\{f_{3}, f_{1}, f_{18}\right\}$ and the third is $\left\{f_{3}, f_{1}, f_{18}, f_{20}\right\}$. The rest may be deduced by analogy. The KNN based classification result is shown in Figure 7.

Figure 7 illustrates that the accuracy curve presents an ascending-stable-descending trend as a whole (red dot line). The accuracy rises firstly (from $61.4 \%$ to $85 \%$ ) and maintains a relatively stable level (around 85\%); then it falls below $80 \%$ primarily due to the features' redundancy. From the fifth to the tenth feature set, the accuracy varies slightly and keeps constant approximately, so the fifth feature set $\left\{f_{3}, f_{1}, f_{18}, f_{20}\right.$, $\left.f_{4}, f_{6}\right\}$ could be considered as a turning point (intersection of the red solid line and the curve), and it is set as the optimal feature set here (the number of features is six). In practice, the first seven features $\left\{f_{3}, f_{1}, f_{18}, f_{20}, f_{4}, f_{6}, f_{16}\right\}$ are selected to construct $C_{7}^{6}$ feature sets to enlarge the scope of tolerance error, and then the derived feature sets are

$$
\begin{aligned}
& F_{1}=\left\{f_{3}, f_{1}, f_{18}, f_{20}, f_{4}, f_{6}\right\}, \\
& F_{2}=\left\{f_{3}, f_{1}, f_{18}, f_{20}, f_{4}, f_{16}\right\}, \\
& F_{3}=\left\{f_{3}, f_{1}, f_{18}, f_{20}, f_{6}, f_{16}\right\}, \\
& F_{4}=\left\{f_{3}, f_{1}, f_{18}, f_{4}, f_{6}, f_{16}\right\}, \\
& F_{5}=\left\{f_{3}, f_{1}, f_{20}, f_{4}, f_{6}, f_{16}\right\}, \\
& F_{6}=\left\{f_{3}, f_{18}, f_{20}, f_{4}, f_{6}, f_{16}\right\}, \\
& F_{7}=\left\{f_{1}, f_{18}, f_{20}, f_{4}, f_{6}, f_{16}\right\} .
\end{aligned}
$$



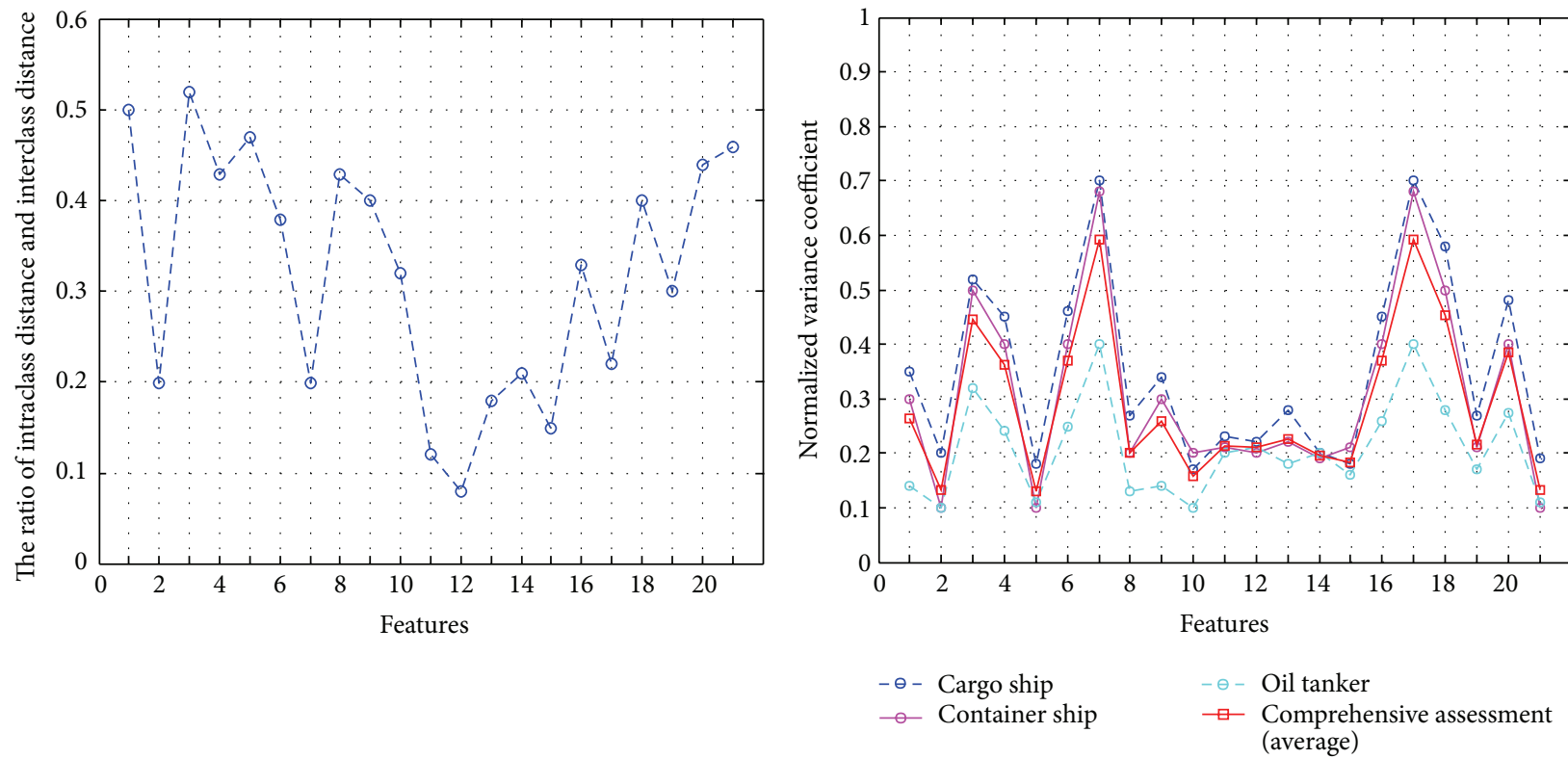

(a)

(b)

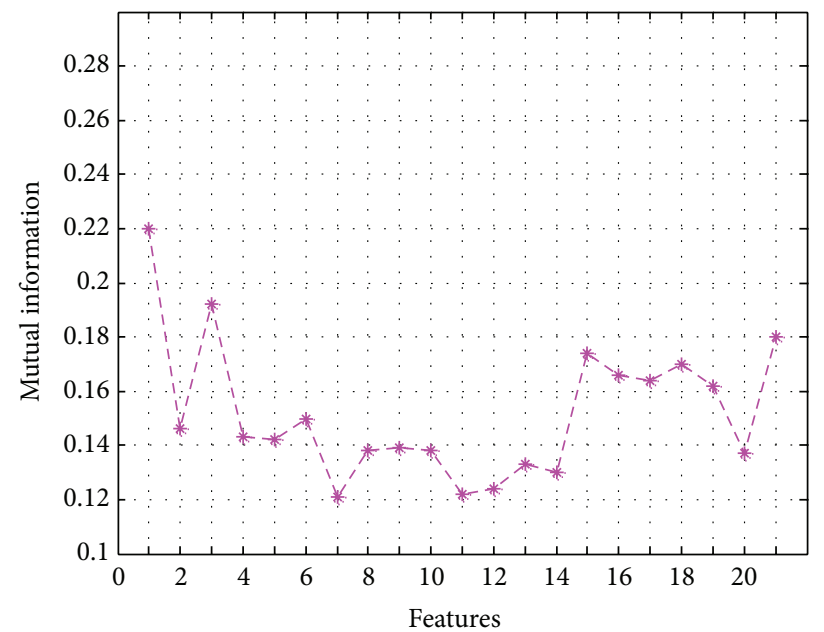

(c)

Figure 5: Analysis of feature attribute. (a) Discriminability (the ratio of intraclass distance and interclass distance), (b) stability (normalized variance coefficient), and (c) BIF (mutual information).

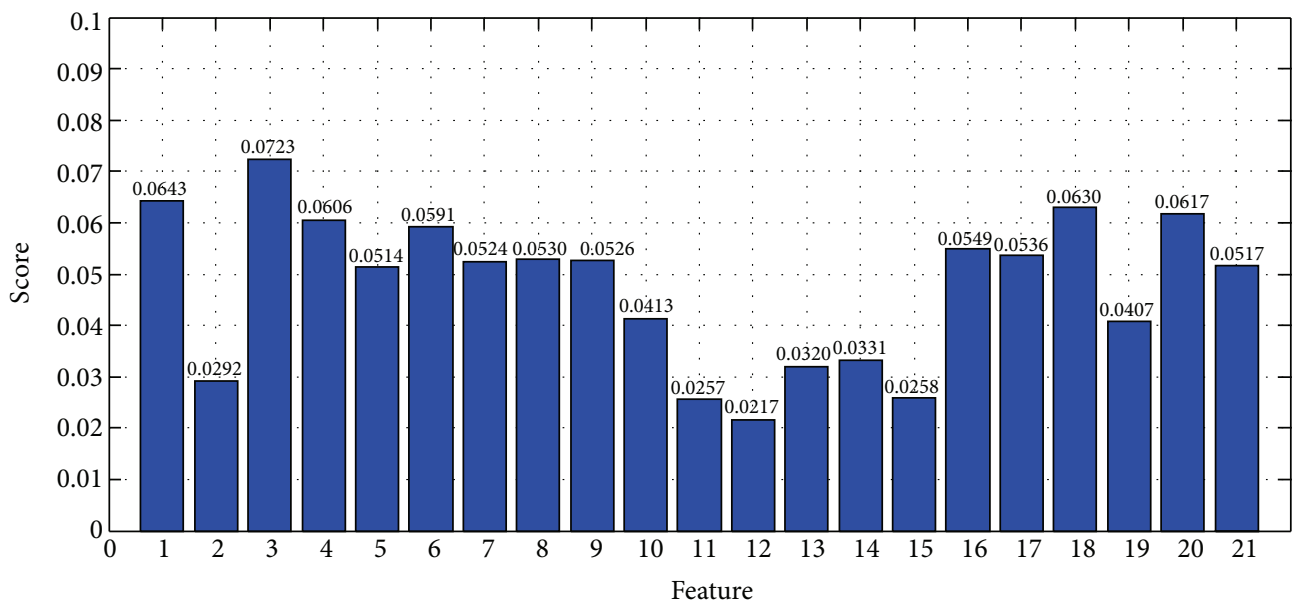

FIGURE 6: Total score for every feature. 


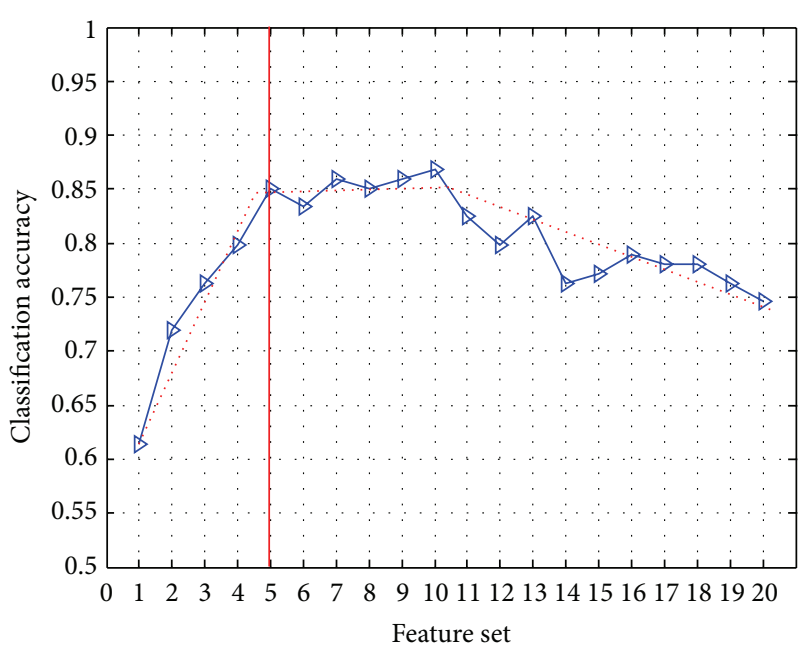

Figure 7: Classification accuracy for the feature sets.

According to the feature scores, the feature sets' priorities are derived:

$$
F_{1}>F_{2}>F_{3}>F_{4}>F_{5}>F_{6}>F_{7}
$$

4.3. Ship Classification Based on Single Classifier. The experiment is carried out based on the test set of ship chips. When a ship chip is input for classification, the feature sets $\left\{F_{n}, n=\right.$ $1 \sim 7\}$ derived above are set as the inputs for KNN classifier (the neighbor parameter $K=3$ in this experiment). In this experiment, $M=7, N=3$. According to the feature sets' priorities derived in the feature selection, the pair-wise comparison matrix can be set as

$$
\mathbf{A}=\left[\begin{array}{cccccccc} 
& F_{1} & F_{2} & F_{3} & F_{4} & F_{5} & F_{6} & F_{7} \\
F_{1} & 1 & 2 & 3 & 4 & 5 & 6 & 7 \\
F_{2} & \frac{1}{2} & 1 & 2 & 3 & 4 & 5 & 6 \\
F_{3} & \frac{1}{3} & \frac{1}{2} & 1 & 2 & 3 & 4 & 5 \\
F_{4} & \frac{1}{4} & \frac{1}{3} & \frac{1}{2} & 1 & 2 & 3 & 4 \\
F_{5} & \frac{1}{5} & \frac{1}{4} & \frac{1}{3} & \frac{1}{2} & 1 & 2 & 3 \\
F_{6} & \frac{1}{6} & \frac{1}{5} & \frac{1}{4} & \frac{1}{3} & \frac{1}{2} & 1 & 2 \\
F_{7} & \frac{1}{7} & \frac{1}{6} & \frac{1}{5} & \frac{1}{4} & \frac{1}{3} & \frac{1}{2} & 1
\end{array}\right] .
$$

The maximum eigenvalue of $\mathbf{A}$ is $\lambda_{\max }(\mathbf{A})=7.2786, \mathrm{CI}=$ $\left(\lambda_{\max }(\mathbf{A})-n\right) /(n-1)=0.0464$. From Table 2 , the value of RI could be acquired $(\mathrm{RI}=1.32)$, and then $\mathrm{CR}=\mathrm{CI} / \mathrm{RI}=$ $0.0352<0.1$, so the consistency of $\mathbf{A}$ is satisfied. The standardization form of the maximum eigenvector is

$$
\mathbf{s}=[0.3086,0.2369,0.1745,0.1221,0.0803,0.0490,0.0286]^{T} .
$$

Then, ship classification using KNN classifier is based on the seven feature sets $\left\{F_{i}, i=1,2,3,4,5,6,7\right\}$. As to each feature set, the comparison matrix for the scheme layer is constructed according to Section 3.3. And then, the comparison matrix for other feature sets could be constituted similarly. The corresponding weighting vectors can be calculated. The total scores for the three kinds (cargo ship, container ship, or oil tanker) can be derived and the final decision (the type of input ship chip) is made.

When the classification decision of one ship chip is made, others are processed one by one based on the same method. Once the classification decisions of all the ship chips are made, the classification accuracy (for each type and the total accuracy) is calculated by the ratio of correct-classified number to the total number. Classification accuracies of the comparisons between the single-feature-set based classifications and the presented AHP-based classification are shown in Table 3. The corresponding diagram of confusion matrix is presented in Figure 8 . KNN classification based on the derived feature sets $\left\{F_{n}, n=1 \sim 7\right\}$ could achieve satisfied performance. Inparticular for the AHP based method, the classification accuracy rates of the cargo and container ship come to $89.1 \%$ and $89.3 \%$ respectively, and the comprehensive performance is superior to other methods with the total accuracy of $87.7 \%$.

In addition, some cases in which the vessel was wrongly classified (take the oil tanker case as an example) are presented in Table 4 and some analysis of the possible causes is also presented. There are three chips whose oil pipe structure could be identified by human operator but not as clear as the ideal ones due to many interferential brighter scattering points around the oil pipe. However, they are all wrongly classified into cargo ships. In these three cases, the third chip is disturbed by strong scattering points badly and none of the chosen feature sets could be classified correctly, so the final decision based on AHP considers $0 \%$ possibility for the oil tanker. From the cases for chips No. 1 and No. 2, we can see that only $F_{3}$ and $F_{5}$ classify the first one into oil tanker, and $F_{2}, F_{3}, F_{5}$, and $F_{6}$ classify the second one correctly. Referring to the detailed features for each set shown in Section 4.2, the possible reasons for these two cases are analyzed below.

$F_{1}$ classifies the first and second one wrongly; however, $F_{3}$ classifies both correctly and $F_{2}$ classifies the second one correctly. The features affecting their performances focus on the features $f_{4}$ (area), $f_{6}$ (shape complexity), and $f_{16}(\mathrm{Hu}$ moment). Considering their detailed performances, $f_{4}$ and $f_{6}$ are more sensitive to the classification of these two chips compared to other features. Actually, the interferential scattering points really affect ships' shape complexity and the accurate extraction of targets' area. Meanwhile, we can see that feature $f_{16}$ might be more preferable to $f_{4}$ or $f_{6}$ for the oil tanker classification although Section 4.2 shows that the latter two are a little more superior in the overall performance. Concluded from the performance of feature set $F_{5}$ (compared to $F_{4}$ ), possibly Hu moment $f_{20}$ is superior to Hu moment $f_{18}$ in oil tanker classification.

Actually ship features are due to the ship scattering, and the relations among them could be considered as being nonlinear, not totally independent. Essentially, the feature set 


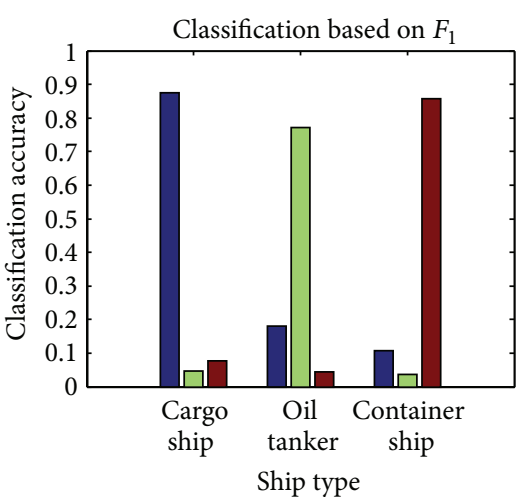

(a)

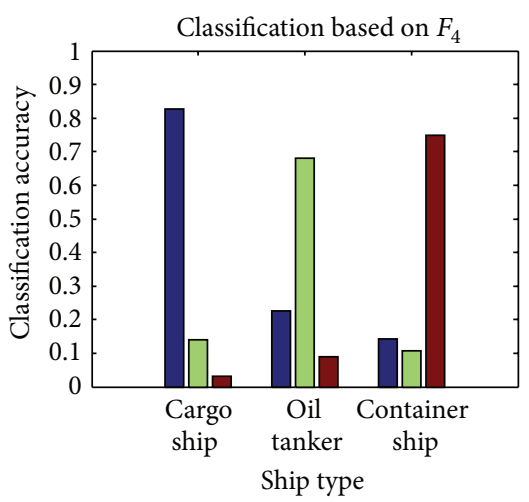

(d)

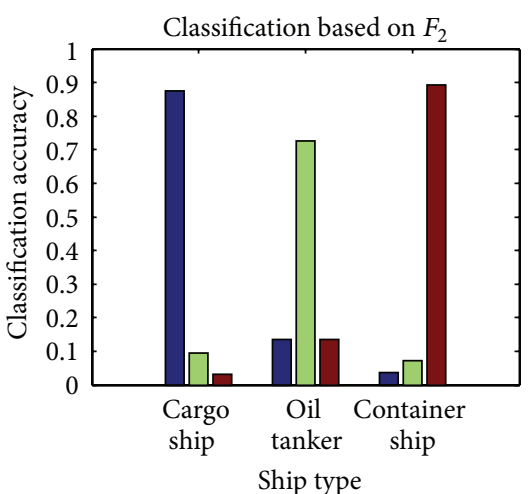

(b)

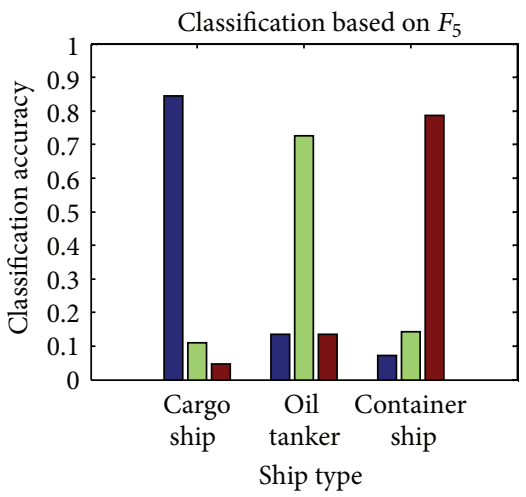

(e)

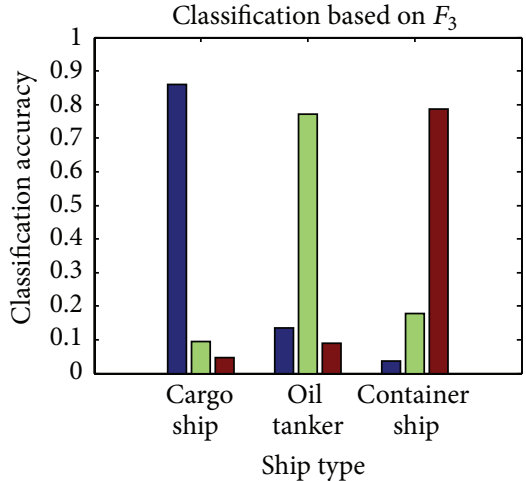

(c)

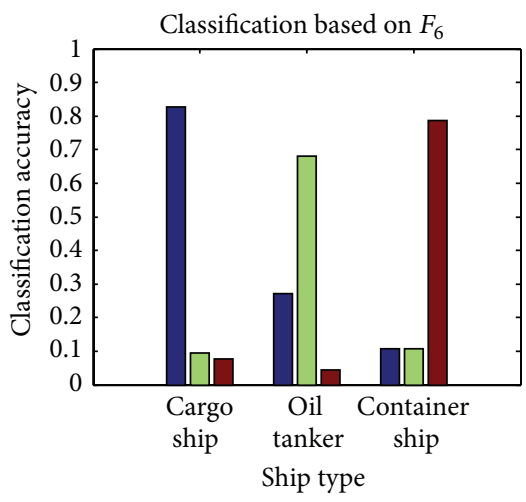

(f)

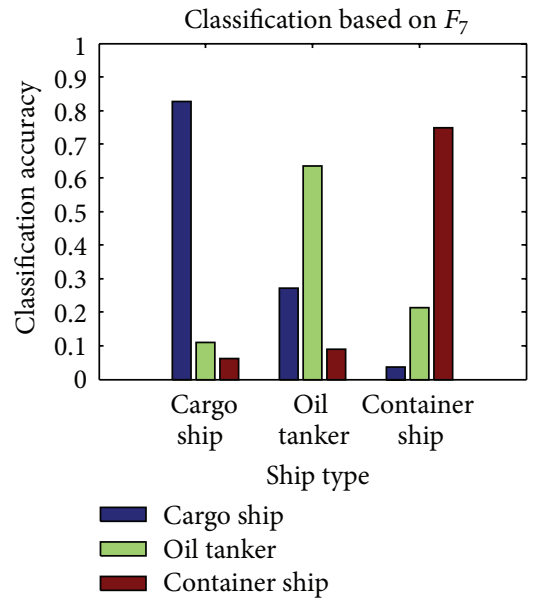

(g)

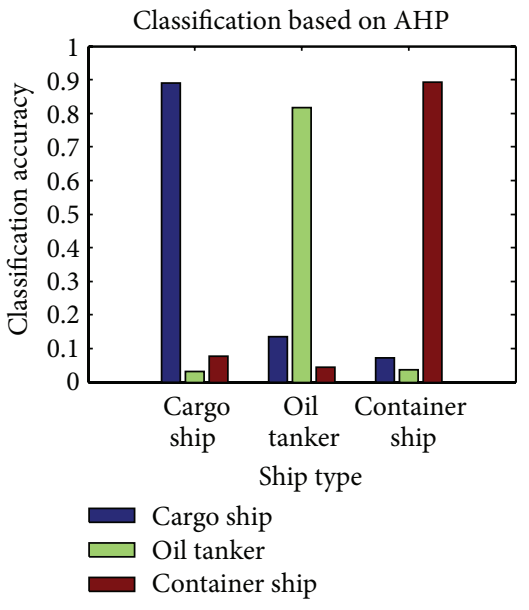

(h)

Figure 8: Diagram of confusion matrix: (a) $F_{1}$, (b) $F_{2}$, (c) $F_{3}$, (d) $F_{4}$, (e) $F_{5}$, (f) $F_{6}$, (g) $F_{7}$, and (h) AHP.

including several features contributes to the classification as a whole. It is quite difficult to clarify their independent contribution clearly.

When it refers to the second ship chip classification, four of seven feature sets are classified correctly, however, $F_{2}, F_{3}$, $F_{5}$, and $F_{6}$ have their own superiorities (in this experiment they are assigned $1 / 2,1 / 3,1 / 5$, and $1 / 6$ ). When the final decision based on AHP is made, the total score of classification into oil tanker is a little less than the score of classification into cargo. Actually, this is only a decision problem, and it just illustrates the possibility of falling within a certain type of ships. When the superiority factors of the feature sets vary proportionally, the results might vary but the conclusions are roughly consistent.

\section{Discussion and Conclusion}

This paper investigates the application of AHP in the feature selection and classification decision. Ship classification based on AHP could make more accurate decisions. It considers various contributions which is beneficial to the final decision 
TABle 3: Ship classification accuracy based on KNN classifier.

\begin{tabular}{lcccc}
\hline Methods & \multicolumn{2}{c}{$\begin{array}{c}\text { Ship type } \\
\text { Oil tanker }\end{array}$} & Container ship & Total accuracy \\
\hline$F_{1}$ & $87.5 \%$ & $77.3 \%$ & $85.7 \%$ & $85.1 \%$ \\
$F_{2}$ & $87.5 \%$ & $72.7 \%$ & $89.3 \%$ & $85.1 \%$ \\
$F_{3}$ & $85.9 \%$ & $77.3 \%$ & $78.6 \%$ & $78.5 \%$ \\
$F_{4}$ & $82.8 \%$ & $68.2 \%$ & $75 \%$ & $80.7 \%$ \\
$F_{5}$ & $84.4 \%$ & $72.7 \%$ & $78.6 \%$ & $78.9 \%$ \\
$F_{6}$ & $82.8 \%$ & $68.2 \%$ & $78.6 \%$ & $77.2 \%$ \\
$F_{7}$ & $82.8 \%$ & $63.6 \%$ & $75 \%$ & $87.7 \%$ \\
AHP & $89.1 \%$ & $81.8 \%$ & $89.3 \%$ & \\
\hline
\end{tabular}

TABLE 4: Wrong classification cases for oil tanker.

\begin{tabular}{|c|c|c|c|}
\hline \multirow{2}{*}{\multicolumn{4}{|c|}{ Oil tanker cases }} \\
\hline & & & \\
\hline$F_{1}$ & $\times$ & $x$ & $x$ \\
\hline$F_{2}$ & $\times$ & $\checkmark$ & $x$ \\
\hline$F_{3}$ & $\checkmark$ & $\checkmark$ & $x$ \\
\hline$F_{4}$ & $x$ & $x$ & $x$ \\
\hline$F_{5}$ & $\checkmark$ & $\checkmark$ & $x$ \\
\hline$F_{6}$ & $\times$ & $\checkmark$ & $x$ \\
\hline$F_{7}$ & $\times$ & $x$ & $\times$ \\
\hline AHP & $x$ & $x$ & $\times$ \\
\hline
\end{tabular}

$\checkmark$ : identified; $\times$ : unidentified.

and optimizes the process of decision. The experiment results illustrated that the AHP-based classification achieves higher accuracy.

Similar to the KNN classifier, ship classification based on Bayes classifier, BP-NN classifier, SVM classifier, and the combination of multiple classifiers could also be implemented using AHP. The comparison matrix for the $N$ classifiers is a $N \times N$ matrix. Because there exit no absolute assessments for the classifiers' performance, the weighting coefficients need to be determined according to the different conditions. For example, when there are less samples comparatively, SVM has better performance, but the classification accuracy of BP-NN is difficult to be improved, so SVM has higher priority than BP-NN. When it is not easy to decide, the coefficients could be set as all ones (with coequal importance). The following steps are similar to ship classification based on single classifier. The only difference is the evaluation criteria. AHP based feature selection and classification are easy to be affected by the subjective factors, so the classification results may vary according to the different evaluations on the importance of criteria. How to establish objective evaluation criteria to constitute reasonable pair-wise comparison matrix needs to be emphasized in the future.

Essentially, ship classification using space-borne SAR imagery is a quite difficult problem to deal with. This paper primarily researches on the decision problems for feature selection and classification. Ship classification is based on scattering characteristics affected by SAR monitoring, environmental conditions, and ship status, such as sea state, atmospheric phenomena, ship speed, and ship orientation. Emphasizing the illustration the AHP decision method concisely, this paper simplifies these experimental conditions properly. These problems are important for accurate classification and need to be considered in the future research. Ground truth could also be retrieved via transponder polls, that is, automatic identification system (AIS) [36], and provides references for the ship classification. Multisource data fusion (including SAR, optical satellite, infrared, coastal radar, and AIS, etc.) would provide more valid references and benefit ship classification [37]. These effective validation approaches are necessary to aid ship classification based on SAR imagery. 


\section{Acknowledgments}

This work is supported by the National Natural Science Foundation of China under Grant 61240058 and the Fund of Innovation provided by Graduate School of National University of Defense Technology under Grant S130401. The authors would like to thank the anonymous reviewers for their very competent comments and helpful suggestions.

\section{References}

[1] S. Brusch, S. Lehner, T. Fritz, M. Soccorsi, A. Soloviev, and B. Van Schie, "Ship surveillance with TerraSAR-X," IEEE Transactions on Geoscience and Remote Sensing, vol. 49, no. 3, pp. 10921103, 2011.

[2] G. Margarit, J. A. B. Milanés, and A. Tabasco, "Operational ship monitoring system based on Synthetic Aperture Radar processing," Remote Sensing, vol. 1, no. 3, pp. 375-392, 2009.

[3] G. Ferrara, M. Migliaccio, F. Nunziata, and A. Sorrentino, "Generalized-K (GK)-based observation of metallic objects at sea in full-resolution Synthetic Aperture Radar (SAR) data: a multipolarization study," IEEE Journal of Oceanic Engineering, vol. 36, no. 2, pp. 195-204, 2011.

[4] F. Nunziata, M. Migliaccio, and C. E. Brown, "Reflection symmetry for polarimetric observation of man-made metallic targets at sea," IEEE Journal of Oceanic Engineering, vol. 37, no. 3, pp. 384-394, 2012.

[5] R. O. Duda, P. E. Hart, and D. G. Stork, Pattern Classification, John Wiley \& Sons, New York, NY, USA, 2001.

[6] S. Musman, D. Kerr, and C. Bachmann, "Automatic recognition of ISAR ship images," IEEE Transactions on Aerospace and Electronic Systems, vol. 32, no. 4, pp. 1392-1404, 1996.

[7] A. O. Knapskog, "Automatic classification of ships in ISAR images using wire-frame models," in Proceedings of 5th European Conference on Synthetic Aperture Radar (EUSAR '04), pp. 953-956, Ulm, Germany, 2004.

[8] M. Vespe, C. J. Baker, and H. D. Griffiths, "Radar target classification using multiple perspectives," IET Radar, Sonar and Navigation, vol. 1, no. 4, pp. 300-307, 2007.

[9] A. O. Knapskog, S. Brovoll, and B. Torvik, "Characteristics of ships in harbour investigated in simultaneous images from TerraSAR-X and PicoSAR," in Proceedings of the IEEE International Radar Conference (RADAR '10), pp. 422-427, Washington, DC, USA, May 2010.

[10] G. Margarit and J. J. Mallorqui, "Assessment of polarimetric SAR interferometry for improving ship classification based on simulated data," Sensors, vol. 8, no. 12, pp. 7715-7735, 2008.

[11] G. Margarit, J. J. Mallorquí, and X. Fàbregas, "Single-pass polarimetrie SAR interferometry for vessel classification," IEEE Transactions on Geoscience and Remote Sensing, vol. 45, no. 11, pp. 3494-3502, 2007.

[12] G. Margarit and A. Tabasco, "Ship classification in single-pol SAR images based on fuzzy logic," IEEE Transactions on Geoscience and Remote Sensing, vol. 49, no. 8, pp. 3129-3138, 2011.

[13] P. Premaratne and F. Safaei, "Ship classification by superstructure moment invariants," in Proceedings of the International Conference on Intelligent Computing, pp. 327-335, Ulsan, Republic of Korea, 2009.

[14] C. M. Pilcher and A. Khotanzad, "Maritime ATR using classifier combination and high resolution range profiles," IEEE Transactions on Aerospace and Electronic Systems, vol. 47, no. 4, pp. 2558-2573, 2011.
[15] R. Touzi, R. K. Raney, and F. Charbonneau, "On the use of permanent symmetric scatterers for ship characterization," IEEE Transactions on Geoscience and Remote Sensing, vol. 42, no. 10, pp. 2039-2045, 2004.

[16] G. Margarit, J. J. Mallorqui, J. Fortuny-Guasch, and C. LopezMartinez, "Exploitation of ship scattering in polarimetric SAR for an improved classification under high clutter conditions," IEEE Transactions on Geoscience and Remote Sensing, vol. 47, no. 4, pp. 1224-1235, 2009.

[17] G. Margarit and J. J. Mallorqui, "Scattering-based model of the SAR signatures of complex targets for classification applications," International Journal of Navigation and Observation, vol. 2008, Article ID 426267, 11 pages, 2008.

[18] F. Wu, C. Wang, H. Zhang, and B. Zhang, "Analysis of polarimetric ship signatures with RADARSAT-2 quad-pol imagery," in Proceedings of the IEEE International Geoscience and Remote Sensing Symposium (IGARSS '12), pp. 5931-5934, Munich, Germany, 2012.

[19] S. S. Lakshmi, S. K. Patra, J. Saibaba, and G. Varadan, "Polarimetric SAR data analysis for identification and characterization of ships," http://www.geospatialworld.net/Paper/Technology/ArticleView.aspx?aid=24512.

[20] M. Dash and H. Liu, "Feature selection for classification," Intelligent Data Analysis, vol. 1, pp. 131-156, 1997.

[21] L. Yu and H. Liu, "Feature selection for high-dimensional data: a fast correlation-based filter solution," in Proceedings of the 20th International Conference on Machine Learning, pp. 856-863, Washington, DC, USA, August 2003.

[22] A. K. Jain, P. W. Duin, and J. C. Mao, "Statistical pattern recognition: a review," IEEE Transactions on Pattern Analysis and Machine Intelligence, vol. 22, no. 1, pp. 4-37, 2000.

[23] T. L. Saaty, "Relative measurement and its generalization in decision making why pairwise comparisons are central in mathematics for the measurement of intangible factors the analytic hierarchy/network process," Review of the Royal Spanish Academy of Sciences A, vol. 102, no. 2, pp. 251-318, 2008.

[24] E. H. Forman and S. I. Gass, "The analytic hierarchy processan exposition," Operations Research, vol. 49, no. 4, pp. 469-487, 2001.

[25] Z. Shen and Q. Wang, "A novel health evaluation strategy for multifunctional self-validating sensors," Sensors, vol. 13, no. 1, pp. 587-610, 2013.

[26] A. H. I. Lee, C. Y. Lin, H. Y. Kang, and W. H. Lee, "An integrated performance evaluation model for the photovoltaics industry," Energies, vol. 5, no. 4, pp. 1271-1291, 2012.

[27] Y. C. Dong, W. C. Hong, H. Y. Xu, and S. Yu, "Selecting the individual numerical scale and prioritization method in the analytic hierarchy process: a 2-Tuple fuzzy linguistic approach," IEEE Transactions on Fuzzy Systems, vol. 19, no. 1, pp. 13-25, 2011.

[28] J. Jin, L. Rothrock, P. L. McDermott, and M. Barnes, "Using the analytic hierarchy process to examine judgment consistency in a complex multiattribute task," IEEE Transactions on Systems, Man, and Cybernetics A, vol. 40, no. 5, pp. 1105-1115, 2010.

[29] W. Pedrycz and M. Song, "Analytic Hierarchy process (AHP) in group decision making and its optimization with an allocation of information granularity," IEEE Transactions on Fuzzy Systems, vol. 19, no. 3, pp. 527-539, 2011.

[30] H. Y. Tsai and Y. L. Huang, "An analytic hierarchy process-based risk assessment method for wireless networks," IEEE Transactions on Reliability, vol. 60, no. 4, pp. 801-816, 2011. 
[31] X. W. Xing, K. F. Ji, H. X. Zou, W. T. Chen, and J. X. Sun, "Ship classification in TerraSAR-X images with feature space based sparse representation," IEEE Geoscience and Remote Sensing Letters, 2013.

[32] M. Teutsch and G. Saur, "Segmentation and classification of man-made maritime objects in TerraSAR-X images," in Proceedings of the IEEE International Geoscience and Remote Sensing Symposium (IGARSS '11), pp. 2657-2660, Vancouver, Canada, July 2011.

[33] X. J. Tian, C. Wang, H. Zhang, and F. Wu, "Extraction and analysis of structural features of ships in high resolution SAR images," in Proceedings of the IEEE 6th International Conference on Radar (RADAR '11), pp. 630-633, Chengdu, China, October 2011.

[34] A. S. M. Shihavuddin, N. Gracias, R. Garcia, A. C. R. Gleason, and B. Gintert, "Image-based coral reef classification and thematic mapping," Remote Sens, vol. 5, no. 4, pp. 1809-1841, 2013.

[35] T. Fritz, M. Eineder, J. Mittermayer et al., "TerraSAR-X ground segment-basic product specification document," CAF-Cluster Applied Remote Sensing TX-GS-FF-3302, 2009.

[36] Z. Zhao, K. F. Ji, X. W. Xing, H. X. Zou, and S. L. Zhou, "Ship surveillance by integration of space-borne SAR and AIS-review of current research," The Journal of Navigation, 2013.

[37] M. Posada, H. Greidanus, M. Alvarez, M. Vespe, T. Cokacar, and S. Falchetti, "Maritime awareness for counter-piracy in the Gulf of Aden," in Proceedings of the IEEE International Geoscience and Remote Sensing Symposium (IGARSS '11), pp. 249-252, Vancouver, Canada, July 2011. 

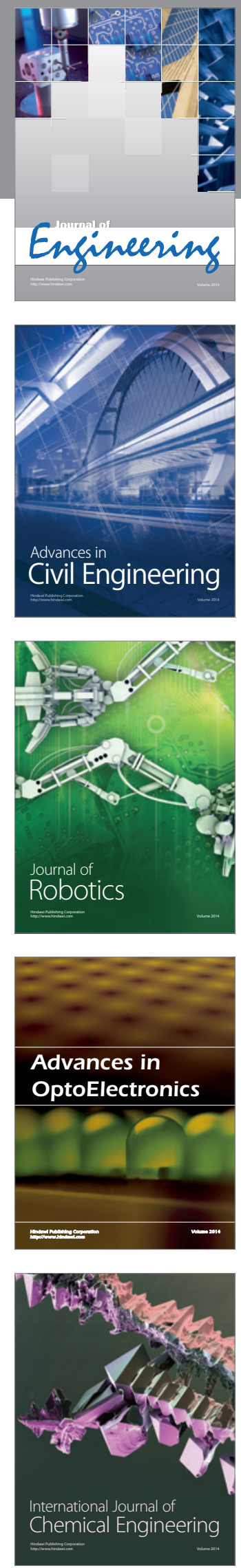

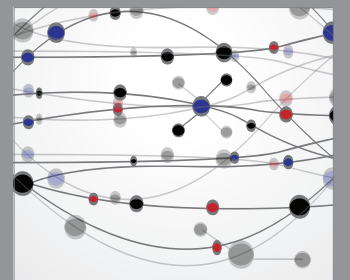

The Scientific World Journal
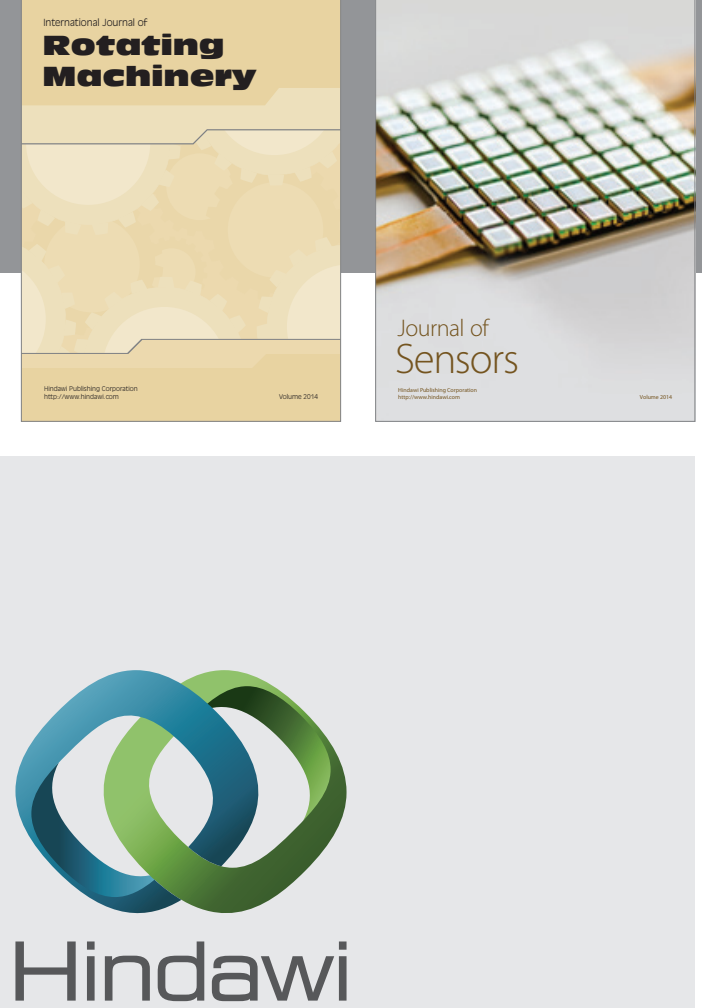

Submit your manuscripts at http://www.hindawi.com
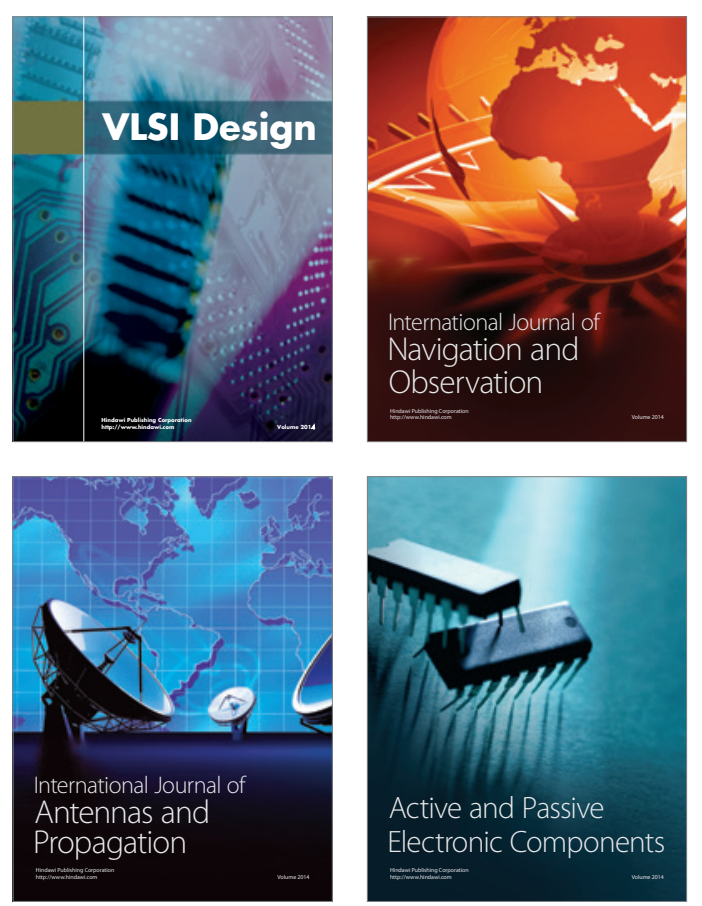
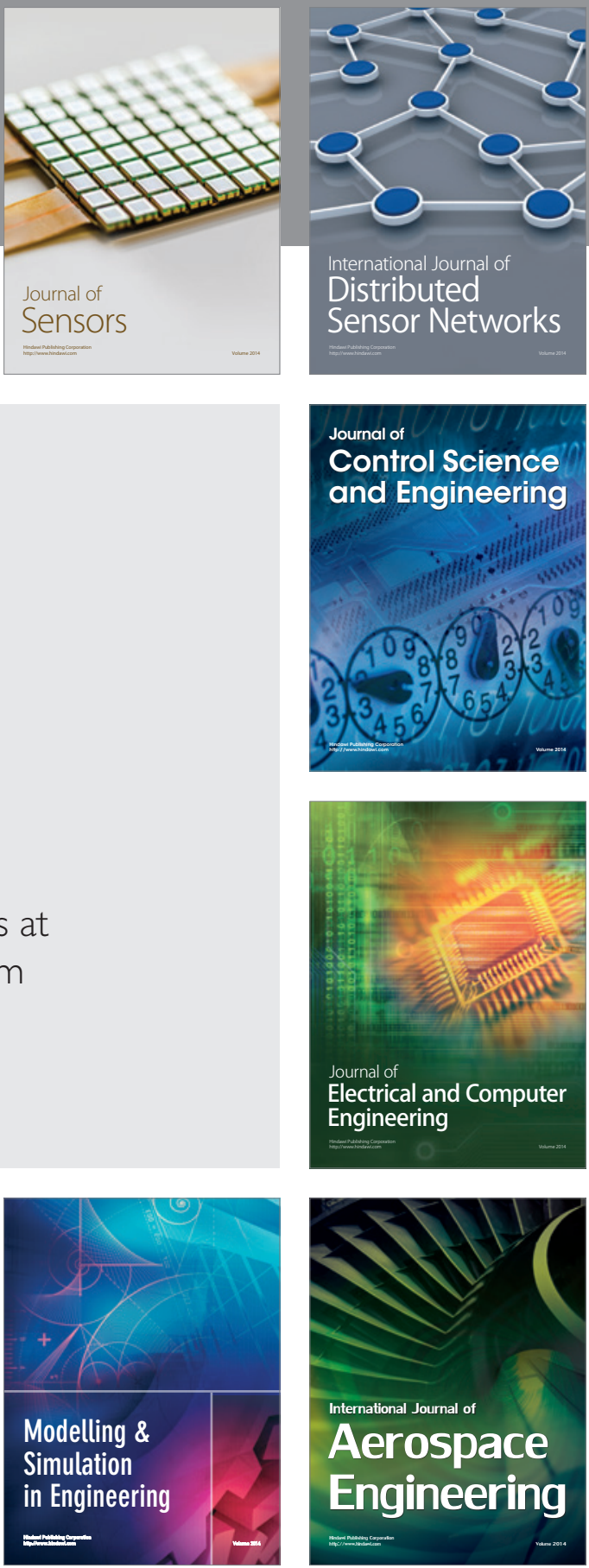

Journal of

Control Science

and Engineering
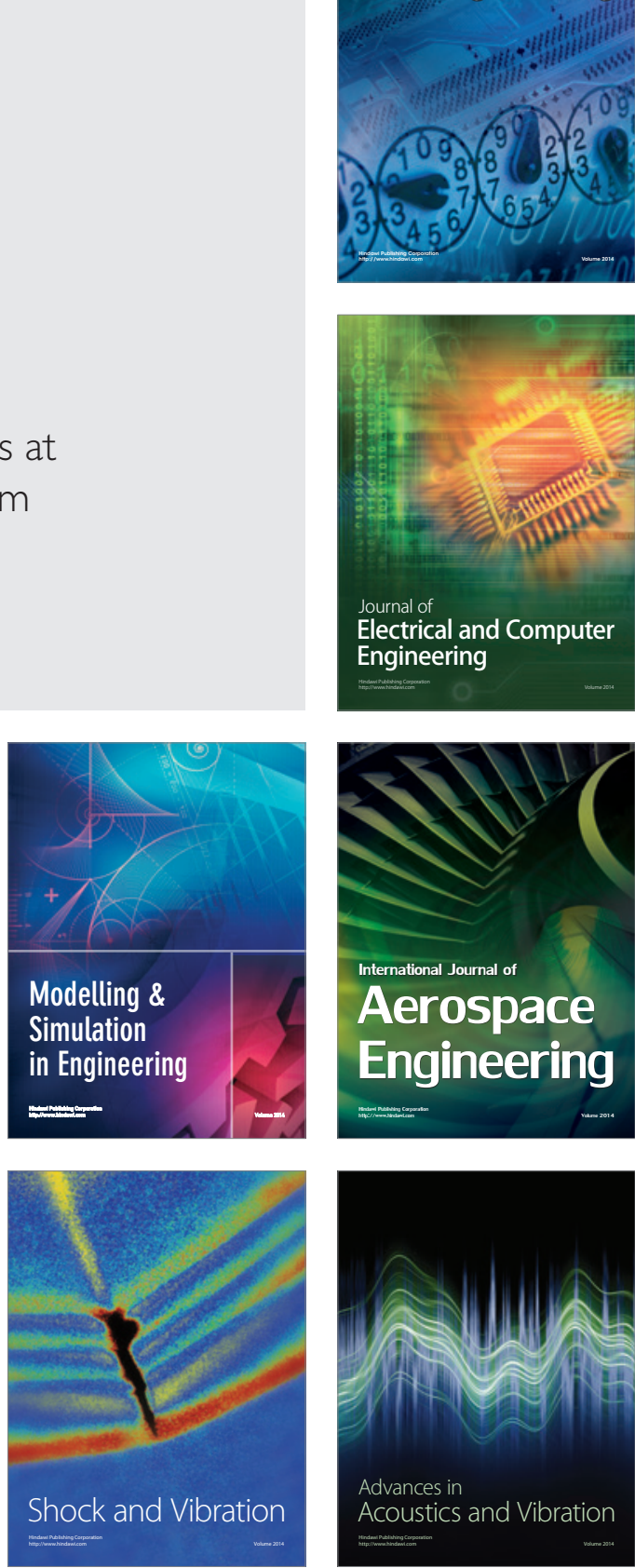